\title{
Dependence of the Electron-Cloud Instability on the Beam Energy
}

\author{
G. Rumolo, ${ }^{1}$ G. Arduini, ${ }^{1}$ E. Métral, ${ }^{1}$ E. Shaposhnikova, ${ }^{1}$ E. Benedetto, ${ }^{1}$ R. Calaga,${ }^{2}$ G. Papotti, ${ }^{1}$ and B. Salvant ${ }^{3}$ \\ ${ }^{1}$ CERN, CH-1211, Geneva 23, Switzerland \\ ${ }^{2}$ Brookhaven National Laboratory, P.O. Box 5000, Upton, New York 11973-5000, USA \\ ${ }^{3}$ EPFL, CH-1015, Lausanne, Switzerland
}

(Received 29 November 2007; published 9 April 2008)

\begin{abstract}
The electron cloud (EC) can be formed in the beam pipe of a circular accelerator if the secondary emission yield (SEY) of the inner surface is larger than 1, and it can detrimentally affect the circulating beam. Understanding the underlying physics and defining the scaling laws of this effect is indispensable to steer the upgrade plans of the existing machines and the design of new ones. The single bunch EC instability (ECI) is shown to be strongly affected by the transverse beam size. Transversely, smaller beams going through an electron cloud generate higher electron peak densities and lower the intensity threshold to make the beam unstable. In particular, since higher energy beams have smaller transverse sizes (for equal normalized transverse emittances), the scaling of the ECI threshold with the beam energy turns out to be surprisingly unfavorable.
\end{abstract}

DOI: 10.1103/PhysRevLett.100.144801

PACS numbers: 29.27.Bd, 29.20.dk, 52.35.Qz

It has been known for many years that secondary electron emission in resonance with an alternating electric field can affect radio frequency (rf) structures [1]. This phenomenon, called the multipactor, leads to exponential electron multiplication, which possibly damages and even destroys the RF device in which it appears. More commonly nowadays, the multipactor has become an obstacle to be avoided for normal operation of particle accelerators, vacuum electronics, radars, and satellite communication devices. The interest to progress on multipactor related issues is therefore broad and extends to several different scientific communities, calling for joint efforts.

In circular accelerators or storage rings operating with positively charged particles, primary electrons are generated via photoemission, gas ionization or particle loss and then they are multiplied by secondary emission, leading to electron-cloud formation. One of the most prominent undesired effects of the electron clouds in these machines is the ECI, where a coherent oscillation of both electrons and beam particles grows from any small initial perturbation of the beam distribution. This instability, which is of the twostream type as studied in plasma physics, can be very fast, since the electron density becomes quickly large due to the closely spaced bunches and to the long survival time of low energy electrons in the beam pipe [2]. The performance of the Super Proton Synchrotron (SPS), the largest ring in the Large Hadron Collider (LHC) injector chain, is currently limited by ECI and transverse mode coupling instability (TMCI). In particular, it is observed that beams become quickly unstable and are deteriorated or lost, if their brilliance at injection is above a certain value. Multibunch operation with high intensity bunches and low transverse emittances causes ECI, which is overcome by operating the ring with large vertical chromaticity after a scrubbing run [3]. High intensity and low longitudinal emittance bunches are affected by TMCI [4]. The upgrade plan of the LHC injector chain foresees the replacement of the present SPS injector, the Proton Synchrotron (PS) ring, by the PS2, with the consequent increase of the injection momentum into the existing SPS from 26 to $50 \mathrm{GeV} / c$ [5]. This is credited to improve the machine performance in many regards (e.g., less space charge and intrabeam scattering, more rigid beams against transverse coupled-bunch instabilities, no transition crossing, lower injection, and capture losses) [6]. Based on the argument that stiffer beams are more stable, also TMCI and ECI would be intuitively expected to relax at higher energy. However, dedicated studies demonstrate that their behavior with energy is not trivial, due to the complex interdependence between various beam parameters. Reference [6] already showed that, if the longitudinal emittance is conserved and the bunch is matched to its bucket, the TMCI threshold is proportional to the slip factor $|\eta|$. Higher relativistic factors $(\gamma)$ situate the machine farther from this limitation, as was expected, but the instability threshold stays finite even when the energy becomes arbitrarily large.

Although the ECI has been long referred to as a type of TMCI [7], the detailed macroparticle simulations and measurements described in this Letter clearly show that its

TABLE I. Parameters used in our study.

\begin{tabular}{lccc}
\hline \hline \multicolumn{1}{c}{ Parameter } & Symbol & Unit & Value \\
\hline Momentum & $p_{0}$ & $\mathrm{GeV} / c$ & $14-450$ \\
Norm. transv. emitt. & $\epsilon_{x, y}(1 \sigma)$ & $\mu \mathrm{m}$ & 2.8 \\
Long. emittance & $\epsilon_{z}(2 \sigma)$ & $\mathrm{eV} \mathrm{s}$ & 0.35 \\
Bunch length & $\sigma_{z}$ & $\mathrm{~m}$ & 0.3 \\
Bunch population & $N$ & & $1.1 \times 10^{11}$ \\
Vertical tune & $Q_{y}$ & & 26.13 \\
Momentum comp. & $\alpha=1 / \gamma_{t}^{2}$ & & 0.00192 \\
Average $e$-cloud density & $\rho_{e}$ & $\mathrm{~m}^{-3}$ & $10^{12}$ \\
\hline \hline
\end{tabular}


mechanism is in reality more complex and subtle. The known scaling laws valid for TMCI do not appear to be directly applicable to the ECI. This Letter summarizes the results of a comprehensive study of the effect of higher injection energy on the ECI, which was carried out via simulations and experiments in the CERN-SPS. Table I shows a list of the essential parameters used for the numerical study (typical LHC-type bunch in the SPS). The main assumptions of our model (reflecting the real situation) are: The longitudinal emittance and the bunch length are kept constant. The momentum spread $\Delta p / p_{0}$ and the matched voltage are readjusted with the energy. The matched voltage scales like $|\eta| / \gamma$. The normalized transverse emittances are constant. Consequently, the transverse beam sizes are scaled as $\sqrt{1 / \gamma}$.

The dependence of the ECI threshold on energy was simulated with the HEADTAIL code [8]. The action of the electron cloud on the bunch is lumped in one or more points along the ring. The $N_{\mathrm{sl}}$ slices of which the bunch is made, successively interact with the electrons (modeled as $N_{e}$ macroparticles and uniformly distributed with zero initial speed in the cross section of the pipe). Each slice sees the electron cloud as deformed by the interaction with the preceding slices. The distortion of the cloud distribution induced by the bunch passing through is the mechanism that couples the motion of subsequent slices and can give rise to instability. To gain an insight into the physical mechanism that determines the type of dependence of the instability threshold on energy, we have first identified the thresholds at different momenta (or energies) assuming a fixed electron-cloud density concentrated in the arcs of the machine (approximately 70\% of the SPS ring consists of dipoles). Figure 1 shows that the ECI threshold is inversely proportional to the momentum under the above assumptions. A very weak dependence on $|\eta|$ seems to be suggested by the two points at 20 and $26 \mathrm{GeV} / c$ (equidistant from transition), which exhibit the same threshold. Our explanation for this anomalous behavior is that, although

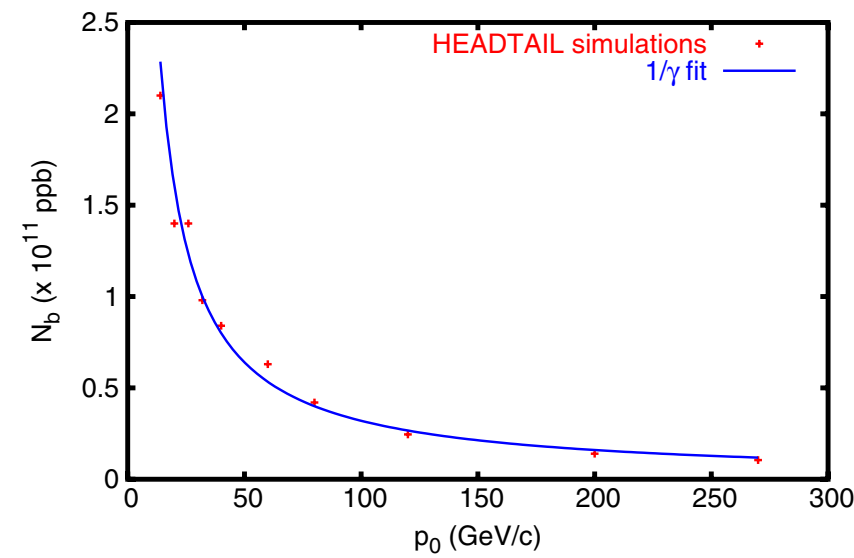

FIG. 1 (color online). Simulated ECI threholds at different momenta, study done with fixed $e$-cloud density. the bunch becomes more rigid at higher energies, and therefore less sensitive to collective effects, it also shrinks transversely, which enhances the effect of the electroncloud pinch. As a result, the "head wake" of the EC (i.e., the response, as electric field averaged over the beam cross section, to a small displacement of the bunch head) contains higher frequencies and amplitudes at higher energies (see Fig. 2). In addition, the matched voltage scales like $|\eta| / \gamma$, causing a decrease of the synchrotron tune far above transition. This translates into a slower longitudinal motion and therefore larger time scales for damping.

This result has been confirmed by PEHTS [9] simulations, which showed similar thresholds to those predicted by HEADTAIL at 40 and $270 \mathrm{GeV} / c$ [10].

The HEADTAIL code has been recently upgraded to allow for more realistic initial distributions of the electrons. A more refined model was needed, because the average electron density over the cross section of the pipe can significantly differ from the local density around the bunch, more directly related to the development of instabilities. HEADTAIL can now load the electron distribution as produced by the buildup code ECLOUD [11] and it uses it for the instability simulation. The result of a scan over a few points extending to $270 \mathrm{GeV} / c$ is displayed in Fig. 3 for a maximum SEY $\left(\delta_{\max }\right)$ of 1.4. The decrease of the threshold with increasing energy is confirmed. Nevertheless, the $1 / \gamma$ decaying law found with the fixed cloud density model changes to a smoother decrease of the threshold with energy, which levels off to the threshold for electron-cloud buildup for momenta higher than $100 \mathrm{GeV} / c$.

To prove that the main cause of the destabilization of the beam at higher energies lies in the smaller transverse sizes, we simulated the ECI at $26 \mathrm{GeV} / c$ for beams with different emittances in both transverse planes. It was found that emittance values about a factor 1.5 larger than nominal are sufficient to stabilize the SPS bunch against ECI (see Fig. 4). This feature is also inherent to ECI and can

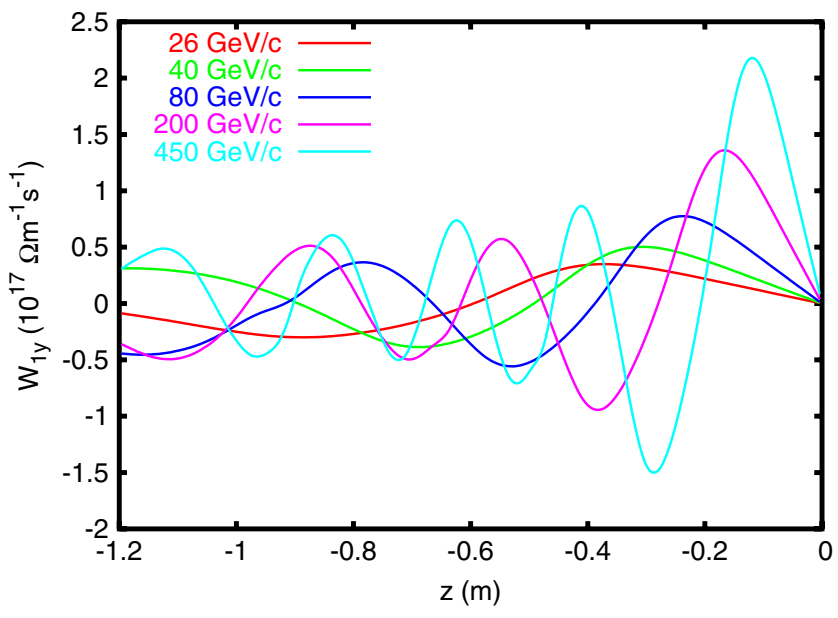

FIG. 2 (color online). EC head wakes at different momenta. 


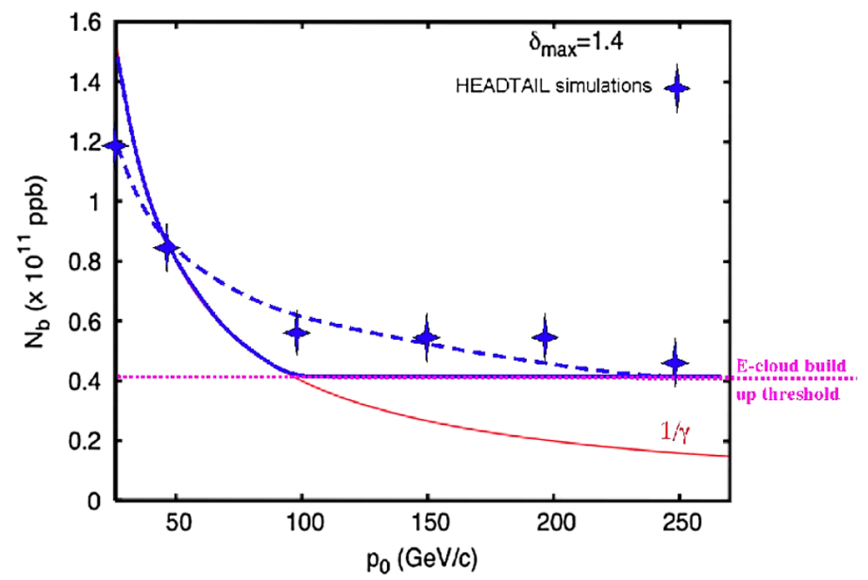

FIG. 3 (color online). Simulated ECI thresholds at different momenta, study done with quasi-self-consistent $e$-cloud distribution.

distinguish it from other types of collective instabilities, which do not depend on $\epsilon_{x, y}$ to the first order (except space charge, which is weak at the energies under consideration in this Letter).

An experimental study to prove the scaling law found by simulations was carried out at the CERN-SPS during the 2007 run. An LHC-type beam made of 4 batches with 72 bunches each, was injected into the SPS at $26 \mathrm{GeV} / c$ during a flat bottom of about $11 \mathrm{~s}$, then accelerated to an intermediate plateau of $55 \mathrm{GeV} / c$ (about $6 \mathrm{~s}$ ) and eventually taken to $270 \mathrm{GeV} / c$ and dumped. The $55 \mathrm{GeV} / c$ flat portion would serve to show that the beam still suffers from ECI at this higher energy. In addition, it could be used to prove that artificially enlarging the beam size would be an efficient tool to suppress this effect. Actually, the observation of the beam behavior at this energy had a twofold

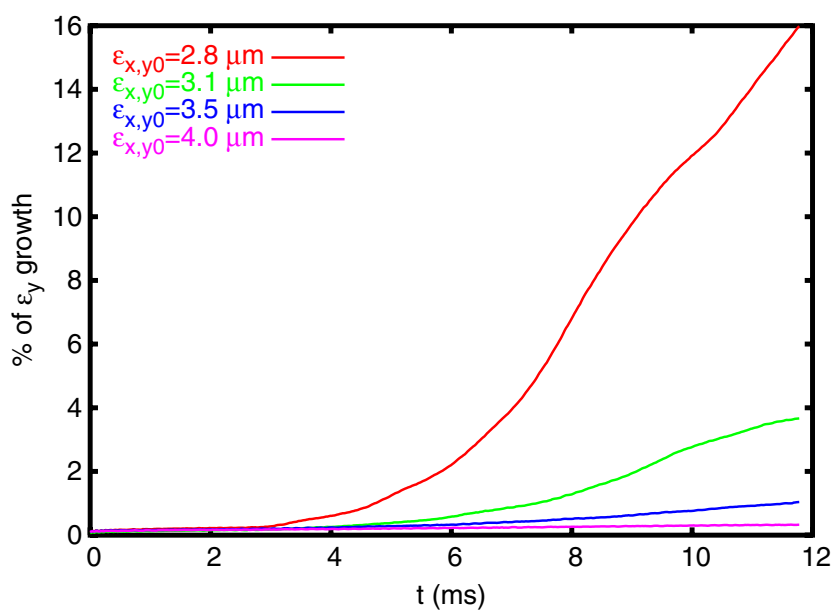

FIG. 4 (color online). Simulated percents of vertical emittance growth due to ECI for LHC-type beams in the SPS having different initial transverse emittances (as labeled). interest, because the value is close to the new injection energy.

After reaching stable conditions with a 4-batch LHCtype beam inside the SPS (tunes $Q_{x}=26.13, Q_{y}=$ 26.185 , and vertical chromaticity $\xi_{y}=0.15$ along the whole cycle), the beam was intentionally driven unstable at $55 \mathrm{GeV} / c$ by reducing the vertical chromaticity to $\xi_{y}=$ 0.05 toward the end of the plateau. The evolution of EC in the machine was monitored through the signal of an EC detector. The ECI manifested itself with uncorrelated coherent vertical motion of the bunches in the tail of the fourth batch and beam loss (see Figs. 5).

The instability was proven to be independent of the operation of the SPS transverse feedback system. The two vertical dampers were switched on and off (alternately and together) with no evident effect on the appearance of the instability. Most of the measurements were taken eventually with both dampers on in order to rule out the onset of coupled-bunch modes. After proving that the ECI appeared at $55 \mathrm{GeV} / c$, it was attempted to suppress it through
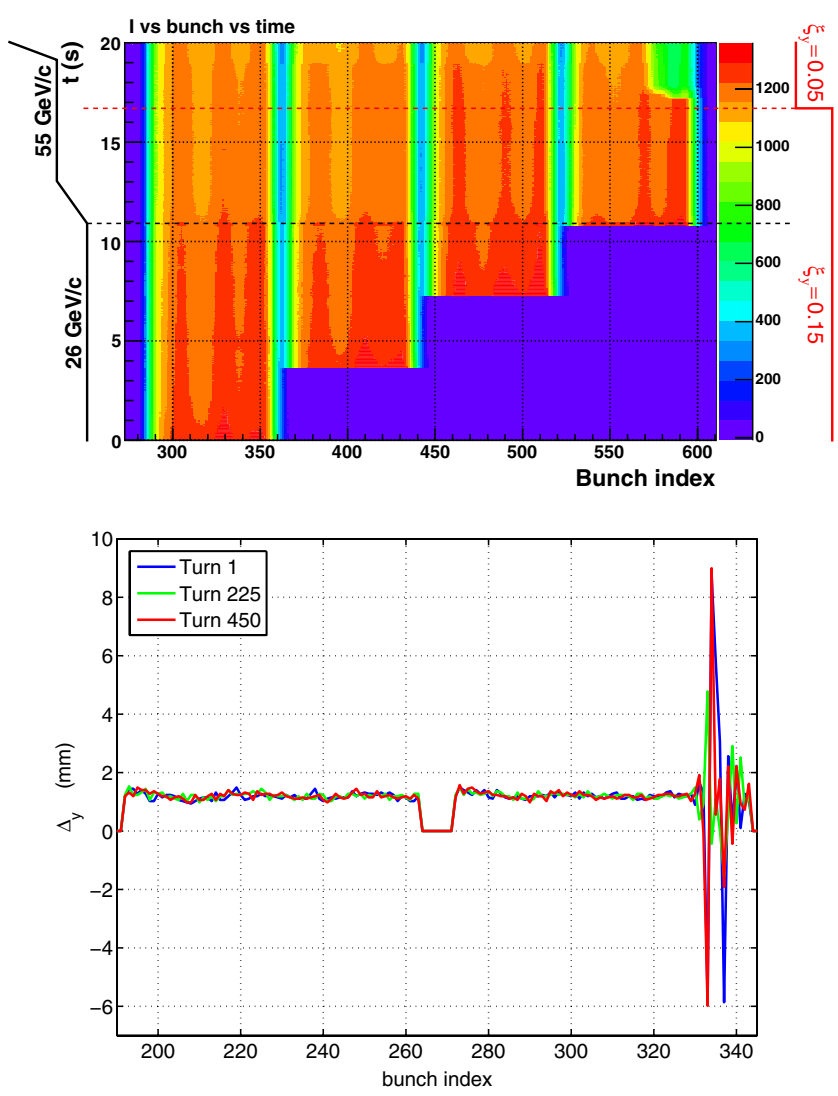

FIG. 5 (color online). Top picture: Evolution of the bunch by bunch intensity with the 4 injections, a small uniform loss at the start of the first ramp, and the final loss at the tail of the fourth batch after lowering the vertical chromaticity. Bottom picture: Three snapshots of the bunch by bunch $\Delta_{y}$ signal in the last two batches. They were captured in an advanced phase of the instability and show that the tail of the fourth batch alone is affected by a vertical single bunch instability. 


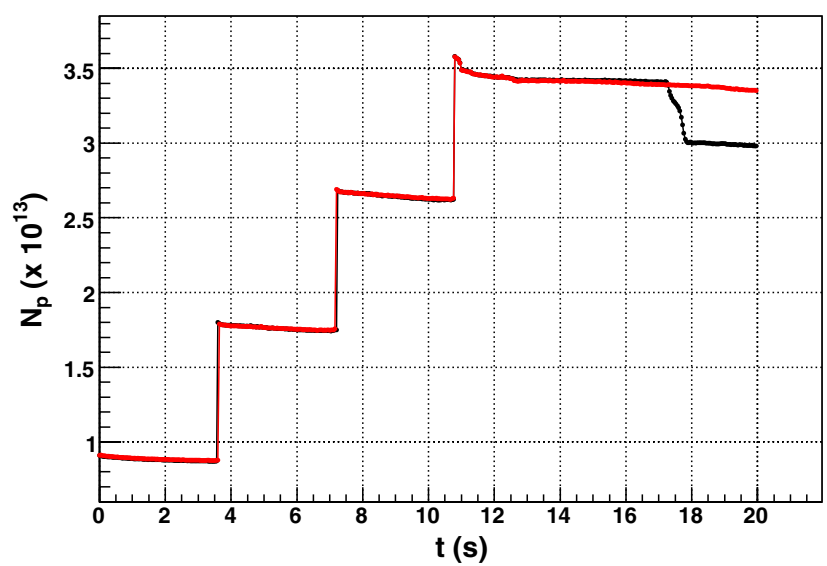

FIG. 6 (color online). Typical beam intensity as a function of time along the cycle. The red trace shows a stable shot when the damper excitation was on, whereas the black trace shows a loss due to a vertical instability occurring with the damper excitation off. In both cases, chromaticity is reduced at $17 \mathrm{~s}$.

transverse emittance blow-up. The longitudinal beam parameters were kept unchanged in this exercise, and they were constantly monitored $\left(4 \sigma_{z}=3 \mathrm{~ns}\right.$ in the flat parts of the cycle). The transverse blow up was introduced at the end of the ramp to $55 \mathrm{GeV} / c$ by means of transverse damper excitation. Testing the method during stable operation with high chromaticity, it was measured that the beam size at the wire scanner location was increased by a factor 2 horizontally and by a factor 1.4 vertically (which would translate in factors of emittance increase of 4 and 2, respectively). As predicted by simulations, the transversely enlarged beam remained stable also when lowering chromaticity to 0.05 . Turning on and off the damper excitation several times, we could observe that the instability would systematically disappear for large beam sizes and reappear for small beam sizes (Fig. 6). In summary, the $55 \mathrm{GeV} / c$ experiment described above shows that one of the bottlenecks for LHC-type beams in the SPS, i.e., the ECI, is still present at higher energy and can lead to beam loss, if it is not suppressed by high chromaticity. Furthermore, it demonstrates the principle that the ECI depends critically on the transverse beam size, as was anticipated by simulations, and can be cured by using beams with larger trans- verse emittances. This evidence can be inferred as an indirect way to prove the physical interpretation of the counterintuitive decrease of the ECI threshold with energy.

In conclusion, both simulations and experiments show that the ECI becomes more severe with increasing beam energy. As a consequence, upgrade plans with higher injection energy for proton machines which suffer from ECI must foresee a program of EC suppression. Promising EC countermeasures exist and their effectiveness is presently under study. They consist mainly of different types of coating, grooved surfaces and clearing electrodes. These techniques normally reduce the effective SEY at the inner pipe surfaces.

The authors are indebted to T. Bohl, W. Fischer, W. Höfle, D. Quatraro, F. Roncarolo, R. Tomás, and F. Zimmermann for fruitful discussion, and to H. Jin and K. Ohmi for code benchmark.

[1] J. R. M. Vaughan, IEEE Trans. Electron Devices 35, No. 7, 1172 (1988).

[2] Proceedings of ECLOUD'04, edited by M. Furman, S. Henderson, and F. Zimmermann (Napa, CA, USA, 19-23 April, 2004), published as CERN Yellow Report No. CERN-2005-001, 2005; and references therein.

[3] G. Arduini et al., Report No. CERN-SL-2001-003-DI, 2001.

[4] G. Arduini, H. Burkhardt and E. Métral, Report No. CERN AB-Note-2003-093-MD 2003.

[5] M. Benedikt, in Proc. of LUMI'06, edited by W. Scandale, T. Taylor, and F. Zimmermann (Valencia, Spain, 16-20 October 2006), published as CERN Yellow Report No. CERN-2007-002 2007.

[6] G. Rumolo, E. Métral, and E. Shaposhnikova, in Proc. of LUMI'06; see above Ref. [5].

[7] K. Ohmi, F. Zimmermann, and E. Perevedentsev, Phys. Rev. E 65, 016502 (2001).

[8] G. Rumolo and F. Zimmermann, Phys. Rev. ST Accel. Beams 5, 121002 (2002).

[9] K. Ohmi and F. Zimmermann, Phys. Rev. Lett. 85, 3821 (2000).

[10] H. Jin and K. Ohmi (private communication).

[11] G. Rumolo and F. Zimmermann, Report No. CERN-SLNote-2002-016-AP, 2002. 